\title{
Auga dos Cebros (Pontevedra, Galicia): un barco del Bronce Final II EN LA FACHADA ATLÁNTICA DE LA PENÍNSULA IbÉRICA (1325-1050 A.C.)
}

\author{
Auga dos Cebros (Pontevedra, Galicia): a Late Bronze Age II Boat in the Atlantic Façade of the Iberian \\ Peninsula (1325-1050 BC)
}

\section{ALFREDO MEDEROS MARTÍN (10)}

Dpto. de Prehistoria y Arqueología. Universidad Autónoma de Madrid. alfredo.mederos@uam.es

\begin{abstract}
RESUMEN:
En Auga dos Cebros (Oia, Pontevedra, Galicia), en el Noroeste de la Península Ibérica, se han localizado tres grabados rupestres de barcos, de los cuales el primero tiene una representación más detallada. Parece ser una embarcación con casco de madera, de unos 16 remeros, que cuenta con un mástil en posición central, con proa y popa elevadas. Una de las opciones, al no indicarse una cubierta superficial, es que se trate de un barco de carga del tipo de Uluburun, ca. 1300 a.C., sin embargo, parece presentar una bancada de remeros por las líneas verticales en el casco, ausentes en el barco de Uluburun. Esto sugiere un barco contemporáneo, pero ya con cubierta superficial, los cuales comienzan con seguridad a partir del Heládico Final IIIB, 1325-1185 a.C., o Chipriota Final IIC, 1300-1190 a.C. en el Egeo. Este tipo de barco no debería sobrepasar el inicio del Bronce Final IIIA o el Protogeométrico en el Egeo, a partir del 1050 a.C., cuando ya eran habituales galeras con numerosos remeros y un tajamar desarrollado, no presente en el barco de Auga dos Cebros.

La frecuentación de la costa de Pontevedra debe estar relacionada con que Galicia, y en particular las rías de Pontevedra, fue la región con estaño aluvial más accesible desde el mar en la Península Ibérica. Por otra parte, ya desde finales del siglo XII a.C. las fuentes clásicas mencionan la fundación del santuario de Melqart en Gadir, ca. 1106 a.C., una de cuyas funciones debió ser facilitar el intercambio del oro y el estaño atlántico.
\end{abstract}

Palabras clave: Edad del Bronce, Galicia, grabados rupestres, barco, comercio de estaño.

\section{Abstract:}

In Auga dos Cebros (Oia, Pontevedra, Galicia), in the Northwest of the Iberian Peninsula, three rock engravings of boats have been located, of which the first has a more detailed representation. It seems to be a boat with a wooden hull, of about 16 oarsmen, that has a mast in central position, with bow and stern raised. One of the options, since no upper deck is indicated, is that it is a cargo ship of the Uluburun type, ca. 1300 BC, nevertheless, seems to display a group of oarsmen by the presence of vertical lines in the hull, absent in the boat of Uluburun. This suggests a contemporary ship, but already with a deck, which begins surely from the Late Helladic IIIB, 1325-1185 BC, or the Late Cypriot IIC, 1300-1190 BC in the Aegean. This type of boat should not exceed the start of the Late Bronze Age IIIA or the Protogeometric in the Aegean, from 1050 BC onwards, when they were already common galleys with numerous paddlers and a developed ram, not present in the boat of Auga dos Cebros.

The frequentation of the Pontevedra coast must be related to the fact that Galicia, and in particular the estuarine inlets in Pontevedra, was from the sea the most accessible alluvial tin region in the Iberian Peninsula. On the other hand, since the late 12th century BC, classical sources mention the foundation of the Melqart shrine in Gadir, ca. 1106 BC, one of whose functions should have been to facilitate the exchange of Atlantic gold and tin.

Key words: Bronze Age, Galicia, rock carvings, boat, tin trade.

Copyright: (C) 2019 Alfredo Mederos. This is an open access paper distributed under the terms of the Creative Commons License, (CC BY-NC-SA 3.0), which permits unrestricted use, distribution, and reproduction in any medium, provided the original author and source are credited. 


\section{INTRODUCCIÓN ${ }^{1}$}

Desde el hallazgo de las pinturas rupestres con barcos de Laja Alta (Cádiz), no ha habido un descubrimiento con representaciones de embarcaciones más importante en la Península Ibérica que el de Auga dos Cebros. Sin embargo, la mayor parte de las referencias sobre estos barcos proceden de menciones puntuales en diversos artículos, sin haber sido tratados en suficiente detalle salvo por Alonso Romero (1995) o Costas y Peña Santos (2005; 2011).

La zona de Santa María de Oia, al S de la ría de Vigo, en la costa de Pontevedra, a medio camino entre Baiona y A Guarda, fue prospectada intensamente por el jesuita portugués padre Eugenio Jalhay, junto con el padre P. Silva, quien antes de la Guerra Civil, desde el monasterio de los jesuitas de Santa María de Oia, descubrió unas 150 estaciones rupestres entre Oia, Mougás, Pedornes y Viladesuso (Jalhay 1926; 1927-29; 1931; 1932). A partir de noviembre de 1992 se realizaron nuevas prospecciones

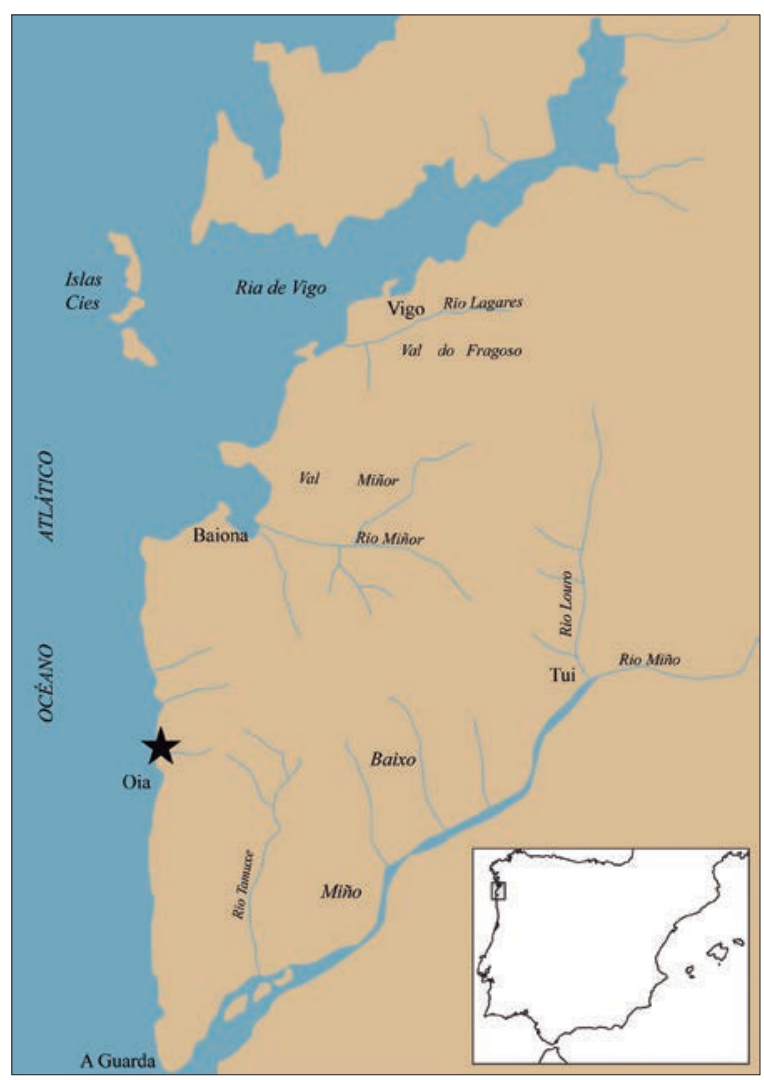

Fig. 1: Costa S de Galicia, con ubicación de Auga dos Cebros (a partir de Costas y Peña 2005: 188 fig.). para volver a localizar muchas de las estaciones mencionadas por el padre Jalhay, descubriéndose la estación de Auga dos Cebros I, en San Mamed de Pedornes (Santa María de Oia, Pontevedra), UTM X: 0510704 Y: 4651412, a finales de ese año o inicios de 1993. La limpieza de unos $20 \mathrm{~cm}$ de tierra que cubría parcialmente la superficie del grabado permitió documentar el primer barco a Pablo Novoa, José Sanromán y Fernando Costas, y fue presentado científicamente ese año en el XXII Congreso Nacional de Arqueología que se celebró en Vigo (Pontevedra) del 17 al 20 de noviembre de 1993 (Costas et al. 1995: 137; Alonso Romero 1995: 131) (fig. 1).

El barco está seriamente dañado en su parte inferior central. Inicialmente se planteó que lo fue con un instrumento metálico (Costas y Peña Santos 2005: 191), sin embargo posteriormente lo relacionaron con un grabado de barco descubierto en Pedornes, que se menciona en un ejemplar de La Voz de Tecla no 156 de 1914, donde se decía que había un tesoro dentro de una roca, marcada con un barco y dos ancletas, en el cual se colocó un cartucho de pólvora y provocó su destrucción parcial, proponiendo que fue la causa del daño en la parte central del barco (Costas y Peña Santos 2006: 278; 2011: 86-87). Otros autores en cambio creen que se trata de desprendimientos $y$ desconchados de la roca soporte (Guerrero 2009: 85), presumiblemente causados por la erosión fluvial (fig. 2).

El grabado de Auga dos Cebros desde su descubrimiento fue calificado de excepcional, sobre todo por el mayor detalle en su representación en comparación con otros grabados previamente conocidos como los de Borna (Pontevedra) (Alonso Romero 1976), siendo ya desde un primer momento encuadrado cronológicamente de una manera muy diversa: como un barco prehistórico (Novoa 1995: 13), un barco de papiros o juncos de la IV Dinastía Egipcia o Imperio Antiguo, es decir, de inicios del Calcolítico Final (Alonso Romero 1995: 140), un barco de fines del II milenio a.C., bien prefenicio: sardo, sículo o tartésico, o bien fenicio (Fábregas y Ruiz Gálvez 1995: 210) o un barco de tecnología mediterránea (Bradley y Fábregas 1996: 108). Las dificultades de su interpretación también ha llevado a evitar pronunciarse por algunos autores por considerarlo de clasificación incierta (Almagro Gorbea 1995: 17; López Castro, com. pers.) o difícil (Rey da Silva 2009; 2015: 389).

Incluso se ha sugerido que el grabado de la primera embarcación no fue realizado por la población local dado su detallismo que exige estar muy familiarizado con ella, atribución foránea incluso adjudicada a los grabados de 


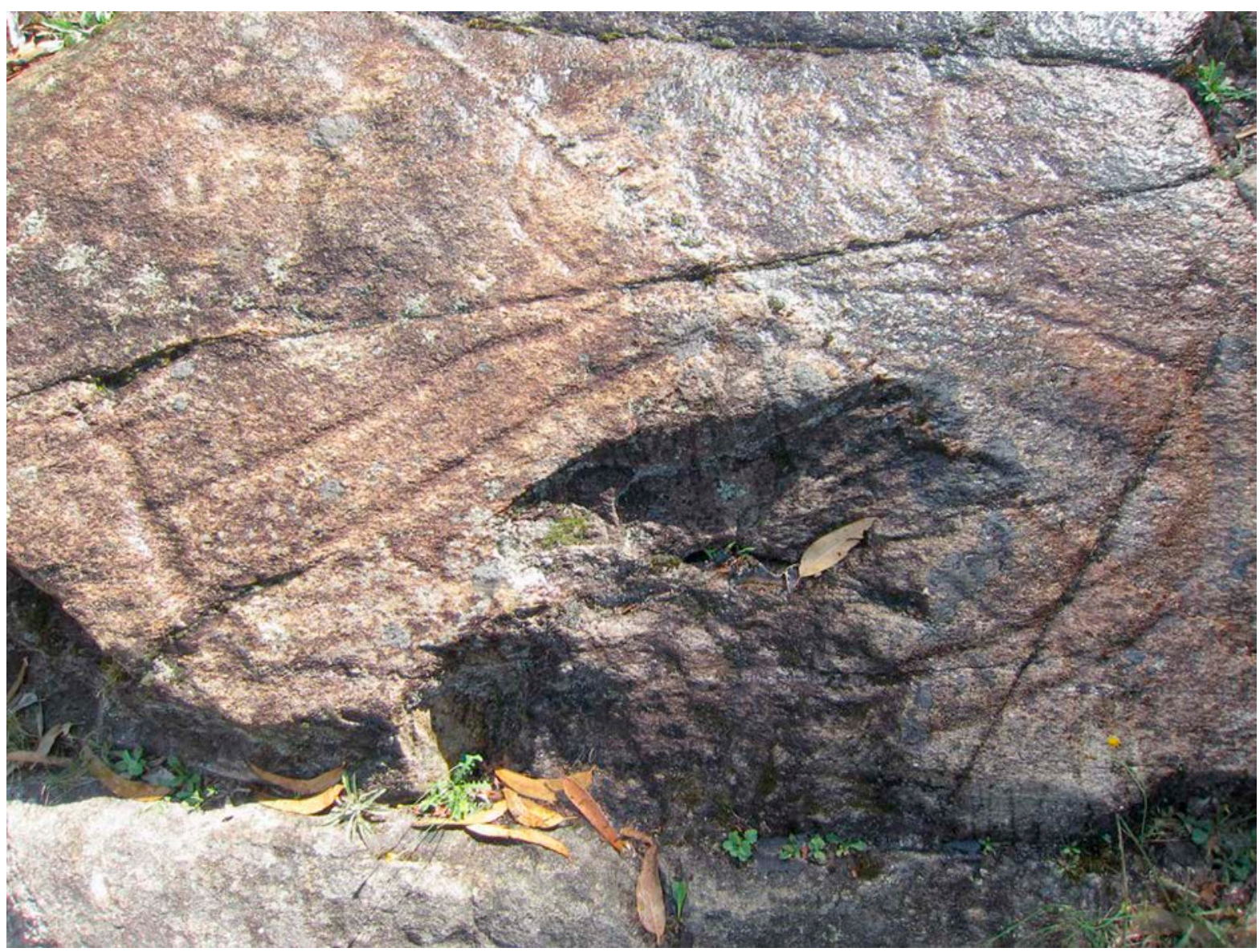

Fig. 2: Primer grabado de barco de Auga dos Cebros (Oia, Pontevedra) con la zona central del casco destruida.

los ciervos, pues la forma de representar a los cuadrúpedos es totalmente desconocida en el corpus de los petroglifos gallegos (Alonso Romero 1995: 138). Estas dudas sobre su autoría las retoma Ruiz-Gálvez (2005: 320), quien plantea tres posibles opciones: pudieron ser dos manos diferentes, indígena en un caso [cérvidos] y mediterráneo en el otro [barco]; se trataría de indígenas estrechamente familiarizados con las características de un barco, seguramente diferente de las tradiciones locales, o bien sería obra de un pequeño grupo de marinos y comerciantes foráneos asentados entre la población local. No obstante, estos grabados en bajorrelieve también se conocen en As Sombriñas en Tourón (Costas y Peña Santos 2011: $101 \mathrm{n}$. 10). Recientemente se ha propuesto que el grabado del barco podría haber sido un exvoto grabado en agradecimiento por el salvamento de la tripulación de un naufragio (Costas y Peña Santos 2011: 280), lo que lógicamente implica que de nuevo se sugiere que fue uno o varios miembros foráneos de la tripulación del barco quienes realizaron el grabado. Personalmente creemos que por las pobres condiciones del fondeadero de Oia, habiendo buenas zonas de fondeo al $\mathrm{S}$ en la desembocadura del río Miño o al $\mathrm{N}$ en la ría de Vigo, difícilmente pudo haber sido obra de marinos foráneos.

\section{LA NAVEGACIÓN POR LA FACHADAATLÁNTICA DE LA PENÍNSULA IBÉRICA Y LA BRETAÑA}

En la navegación atlántica el problema principal es la ruta de $\mathrm{S}$ a $\mathrm{N}$ desde Andalucía hacia Galicia. Esta navegación ascendente por la costa portuguesa no es fácil porque dos tercios del año predominan los vientos del NNW, $\mathrm{N}$ y NNE, en particular, entre mayo y septiembre. Sólo entre noviembre y marzo prevalecen los vientos tropicales de tercer cuadrante, $\mathrm{S}, \mathrm{SO}$ y O, principalmente del SO 
(Riudavets 1867: 15-16), precisamente durante la peor época para navegar a vela que es entre noviembre y febrero. Esto podía alargar el trayecto de ascenso hasta uno o dos meses como recoge este autor, La navegación más molesta que se hace sobre las costas de Portugal y Galicia es la de S. a N. en tiempo de nordestes, y esto suele ocurrir durante todo el verano con leves interrupciones, mayormente en aquellos años que los navegantes califican con el nombre de años de nortes. Se han visto bastante ejemplos de tardar un buque de vela, de uno a dos meses para trasladarse desde el cabo de San Vicente a la ría de Ferrol. Cuando los nortes vienen acompañados de mar, se hace más dilatada la navegación, porque es un aumento de contrariedades que experimenta el navegante, y le obliga a permanecer días y semanas al abrigo del cabo de San Vicente, de Setúbal, Cascais, Peniche, etc., y es raro el buque de cabotaje que en semejante estación no recorra todos estos abrigos (Riudavets 1867: 54-56).

Este trayecto ascendente se podría realizar en apenas cinco días con una velocidad de unos 5 nudos, pero en condiciones normales debió precisar habitualmente en torno a 15 o 20 días y velocidades no superiores a 2,5 nudos, orientándose hacia el Norte con la estrella polar y manteniendo a la derecha la costa, a la vista, pero a cierta distancia. En la derrota o trayectoria debió realizar bordadas contra el viento de unas 3 horas, primero en dirección NE hacia la costa durante el día y luego cambiando a dirección NO, separándose de la costa durante la noche para evitar los peligros de acercarse a ella (com. pers. López Castro).

En cambio, el verano era ideal para realizar una navegación de retorno hacia Andalucía o el Mediterráneo, $L a$ navegación (...) de N. a S., se hace con sumo desahogo en verano, porque es la estación de los nortes, y puede navegarse a longo de costa. No así en invierno y con vendaval, porque se tiene que luchar con viento de proa, sucio, acompañado de mucha mar y corriente contraria (Riudavets 1867: 54).

En la ruta de navegación hacia Bretaña, debían aprovechar los vientos del tercer y cuarto cuadrante existentes entre octubre y junio, por lo que navegarían hacia el $\mathrm{N}$ en primavera, para regresar también durante el verano cuando predominan vientos de primer cuadrante, con rumbo de S a SE (Maciñeira 1947: 312-313).

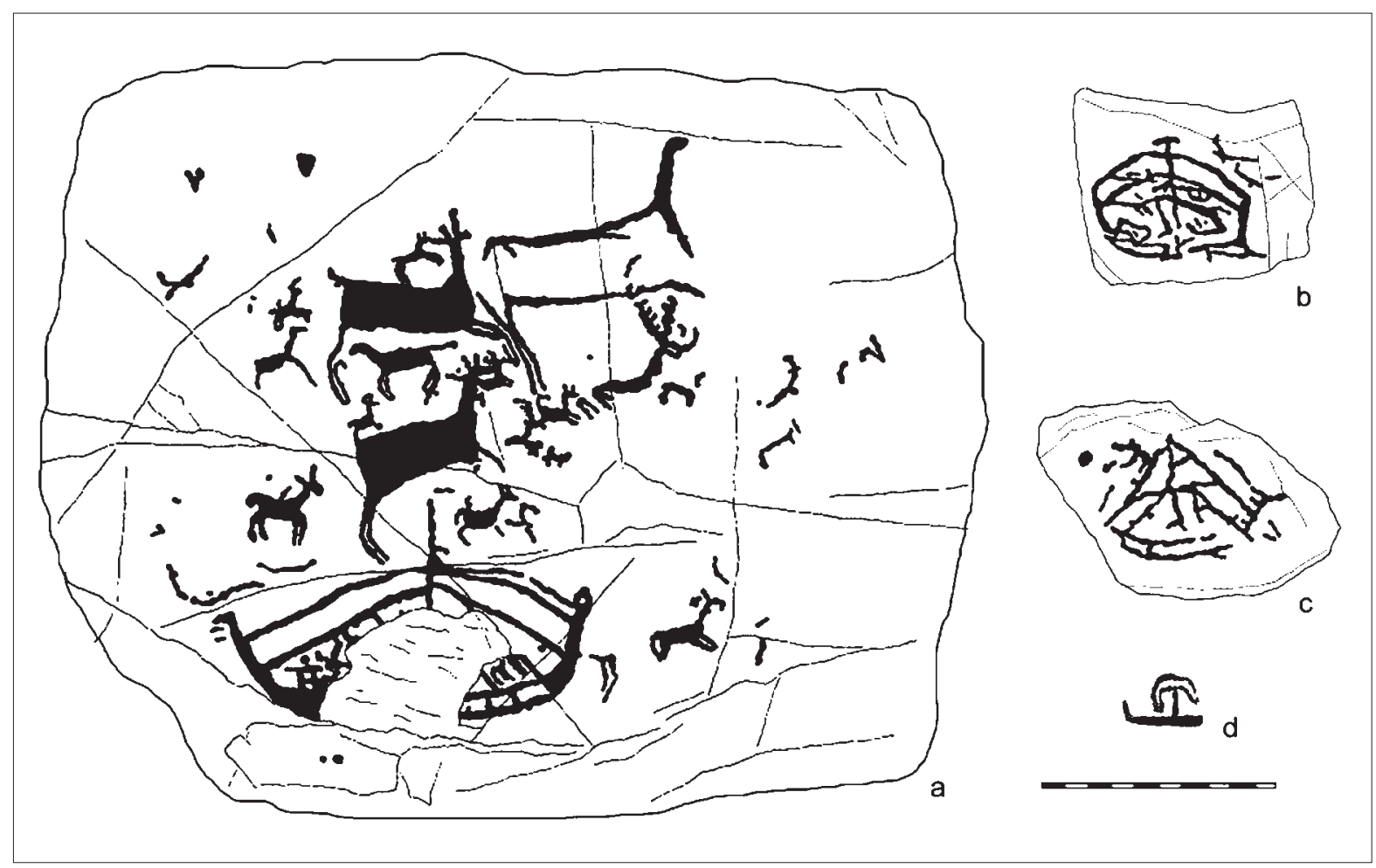

Fig. 3: Grabados de los tres barcos de Auga dos Cebros (Mielke y Schuhmacher 2011: 77 fig. 4). 


\section{LOS BARCOS 2 Y 3 DE AUGA DOS CEBROS}

Ya en la primera publicación se difundió un calco de una segunda embarcación, más pequeña, 0,69 m, y mucho menos precisa en el dibujo del primer barco (Costas et al. 1995: 135 fig. 2f), que años después fue descrita en detalle y denominada O Viveiro IV, sobre un panel de 1,50 x 0,80 m, situado próximo ascendiendo por el riachuelo desde Auga dos Cebros hacia el NO (Costas y Peña Santos 2005: 191, 200-201, 202 fig. y fot.). Su interpretación es compleja, presentando un casco ligeramente curvo, mástil central y presumiblemente uno y quizás dos cabos hacia la proa y lo mismo hacia la popa (fig. 3).

En esta embarcación, Ruiz-Gálvez (2005: 319) cree ver un espolón en el lado izquierdo del grabado, pero trabaja con un calco de la publicación de 1995 que Costas y Peña modificaron en 2005. También trabajando con el mismo calco, Guerrero (2009: 86) asocia dos trazos con un remedo de tajamar.

Un tercer barco se localizó después de una serie de lluvias torrenciales en octubre de 2006, a la derecha del panel de la primera embarcación, denominado Auga dos Cebros I barco 2 por su mayor proximidad, lo que implica que el segundo barco descubierto sería Auga dos Cebros barco 3 u O Viveiro IV. Presenta unas menores dimensiones, $0,55 \times 0,58 \mathrm{~m}$, menos detalles y difícil interpretación. Muestra un mástil central, y tres cabos hacia la proa y tres hacia la popa, con roda y codaste curvos y elevados, mientras la proa parece presentar un tajamar (Costas y Peña Santos 2006: 287-290, 288 fot, 289 fig. 1-2; 2011: 232-233, 234 fig. 1). Este es el detalle naval más interesante porque no es visible en el primer barco. También se sugiere la presencia de una cofa en lo alto del mástil (Costas y Peña Santos 2006: 290; 2011: 232). No obstante, nos vamos a centrar en la primera embarcación que es la que permite realmente un análisis más seguro (fig. 4).

\section{EL PRIMER BARCO DE AUGA DOS CEBROS}

Se trata de una escena donde una gran manada de ciervos se dirige en dirección SE, salvo uno de ellos en dirección NO, de los cuales tres están representados a gran tamaño, quizás los adultos, siendo el mayor de 0,90 x 0,90 m (Costas y Peña Santos 2005: 192). Corren hacia la derecha, mientras en la parte inferior el barco parece navegar en similar dirección. El tipo de grabado en bajorrelieve

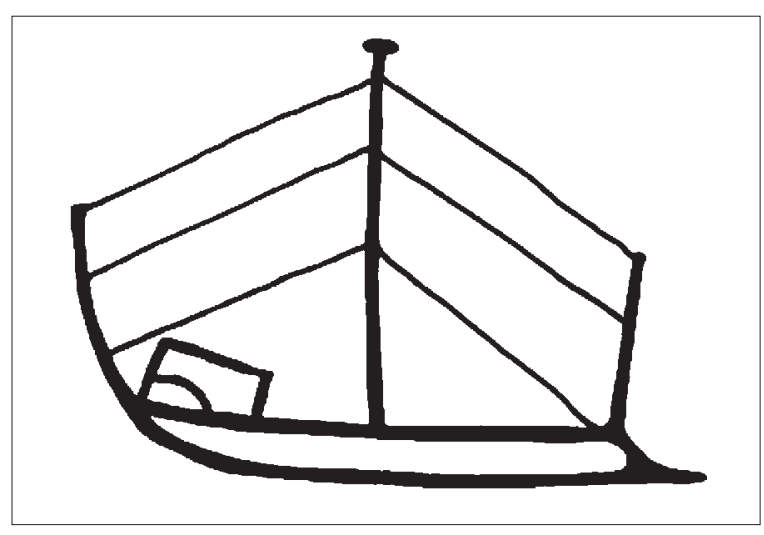

Fig. 4: Propuesta de reconstrucción del segundo barco de Auga dos Cebros con tajamar, según Costas y Peña (2006: 280 fig. 2).

no parece ser diferente entre los motivos principales de ambas composiciones y el mástil del barco además se aproxima al vientre de uno de los ciervos, por lo que quizás los ciervos corren en lo alto de la costa, mientras el barco va costeando el litoral en dirección $\mathrm{S}$, descendente.

Como el grabado se encuentra a $1 \mathrm{~km}$ del mar, a $98 \mathrm{~m}$ snm, junto al denominado río o regato Vilar que desemboca en el Porto de Ribas Altas, y es inundado en ocasiones por el riachuelo que pasa a la derecha del panel cuando hay lluvias intensas (Costas y Peña Santos 2006: 190; 2011: 94 fot. 1), adquiere más sentido la posición inferior en la composición del barco, que puede tocar agua del riachuelo en el punto de finalización del panel cuando el cauce transporta más agua y se circula bordeando parcialmente algunas rocas inmediatas.

La primera descripción náutica precisa fue realizada por Alonso Romero (1995: 138). El barco tiene una eslora o longitud máxima de $1,42 \mathrm{~m}$ con un mástil de $0,65 \mathrm{~m}$. La roda de la proa, que siempre es la más próxima al mástil, está separada $0,54 \mathrm{~m}$, tiene $0,56 \mathrm{~m}$ de altura y termina en un remate circular. El codaste de la popa está ubicado a $0,70 \mathrm{~m}$ del mástil, tiene $0,55 \mathrm{~m}$ de altura y acaba en un remate en forma de martillo según Alonso Romero (1995: 142 fig. 3) o con saliente según Costas, Novoa y Sanromán (1995: 135 fig. 1). Ambos remates son considerados prótomos de ave por Ruiz-Gálvez (1995: 319), aunque posteriormente sólo habla de prótomo de animal sin especificar (Costas y Peña Santos 2005: 210; Guerrero 2009: 85; Ruiz-Gálvez 2013: 276). Debe señalarse que nuestro codaste, siempre el lado más distante al mástil, es considerado la roda por Guerrero (2009: 85), o con más reservas por Costas y Peña Santos (2005: 211), por 
la identificación del prótomo, y Guerrero no descarta que en la zona rota del grabado pudo estar representado un tajamar o proyección a ras del agua. Otra cuestión, que no podemos obviar, e incluso sería más lógico, es que haya un error visual del grabador y haya colocado el mástil ligeramente desplazado hacia la derecha cuando debía ser más central o moverse ligeramente a la izquierda, que si tenemos en cuenta la distancia a la que se vería el barco, tampoco sorprende. Del mástil parten dos cabos hacia la roda de la proa o estays y un cabo hacia el codaste de la popa, pero como es más frecuente que sean dobles en la popa, pues es donde el viento ejerce su fuerza en la vela, puede apoyar, como señala Guerrero (2009: 86), una proa en el lado izquierdo.

Dos trazos inferiores que se dirigen también hacia la roda y codaste han sido considerados una duplicación de los cables de estay y burda según Alonso Romero (1995: 138-139). En cambio, estos dos cabos inferiores que van también hacia la roda y el codaste son considerados por Guerrero (2009: 86) un toldo de una estructura para resguardarse dentro de la nave. También así es identificado por Costas y Peña Santos (2005: 209, 213 fig.), pero en una posición inferior, e incluso sugieren la presencia de tripulantes. Más recientemente identifican una posible

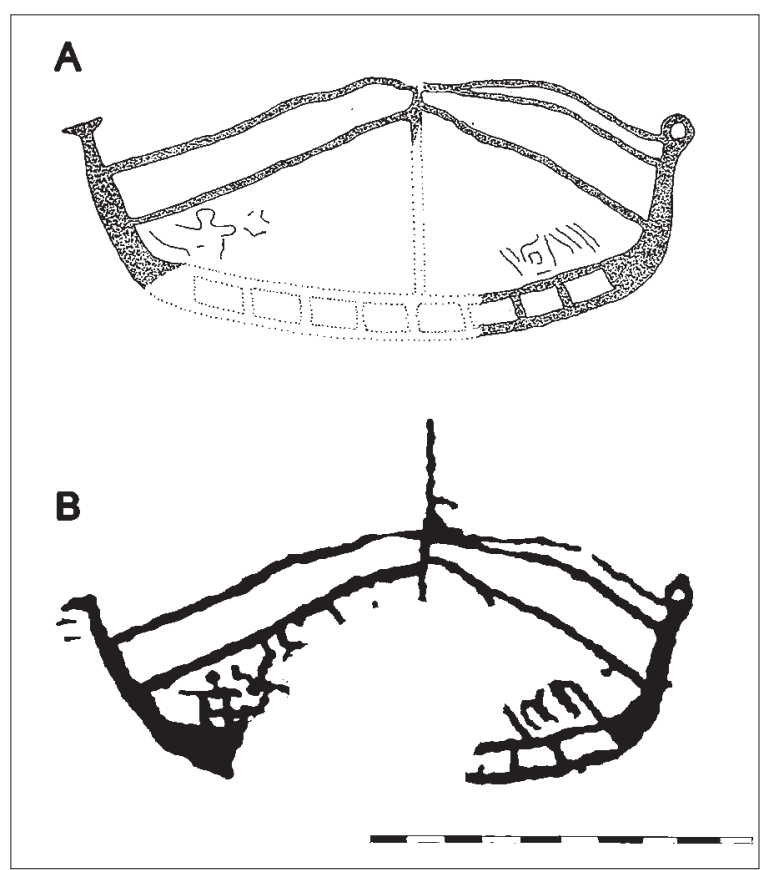

Fig. 5: A: Calco del primer barco de Auga dos Cebros según Alonso (1995: 142 fig. 3). B: Calco del primer barco según Costas y Peña (2005: 213 fig.). plataforma o figura antropomorfa a la izquierda y tripulantes o caracteres alfabéticos a la derecha (Costas y Peña Santos 2011: 106 fig. 2, 107). En cualquier caso, es presumible la existencia de una estructura de piel o madera dentro del barco para protegerse la tripulación de las inclemencias del tiempo, la maresía y las tormentas marinas.

No se representa la verga que sostendría la vela (Alonso Romero 1995: 138), que se sugiere hipotéticamente que sería cuadrada (Costas y Peña Santos 2005: 211). Aunque no se ha planteado, no cabe descartar que la ranura natural de la roca que separa al barco del gran cérvido fuese utilizada por el grabador a modo de verga y por eso no se representase. Si bien en el calco no queda tan claro (fig. 3), sí se aprecia que la ranura natural o verga que sostendría el velamen, pasa justo donde convergen los cables de estay y burda, y en la foto se observa que el surco natural de fisura de la roca es relativamente profundo (fig. 2).

Las medidas del barco son algo diferentes según Costas y Peña Santos (2005: 194-195; 2011: 107), 1,40 m de eslora, 0,95 m de mástil, el cambio más significativo cuando se hizo un nuevo calco en 2005 al prolongarlo, y 0,55 m de altura tanto en la roda como en el codaste (fig. 5).

En conjunto, se trata de un barco con casco curvo caracterizado por tener una proa y popa muy elevadas, adecuado para la navegación en el Atlántico y hacer frente al oleaje. Algunos de estos detalles ya los señalaba Julio César (Bell. Gall. III, 13, 2) como fundamentales en los barcos que transitaban el Atlántico, la proa, muy levantada, y asimismo la popa, acomodadas a la magnitud de las olas y borrascas.

\section{LA HIPÓTESIS DE UN BARCO DE JUNCOS DEL CALCOLÍTICO FINAL}

La propuesta de Alonso Romero resulta coherente con su trayectoria investigadora desde que asoció los posibles grabados de Borna (Meira, Pontevedra), con cascos de cuero y unas proyecciones en la proa y la popa de mimbre, que lo vinculaban con un barco predinástico del Sur de Egipto (Alonso Romero 1976: 111, 116 fig. 30a-b, 119), asignable al Calcolítico Inicial, 3600-3100 a.C., aunque en la actualidad se inclina por situarlo en la Edad del Bronce (Alonso Romero 2011: 104). Una cronología antigua también es propuesta por Guerrero Ayuso (2009: 84) que lo sitúa en el Neolítico Final o Calcolítico, mientras que Pereira y Arruda (2017: 197) también se inclinan por una fecha de la Edad del Bronce. 
Fig. 6: A: Reconstrucción del pecio de Uluburun (Turquía) de $c a$. 1305-1300 a.C. a partir de la distribución del casco de madera y del cargamento (Pulak 2008: 291 fig. 92). B: Reconstrucción tridimensional del barco con su cargamento (Pulak 2008: 293 fig. 94). C: Reproducción moderna del barco de Uluburun con calafateado negro en la parte inferior del casco.

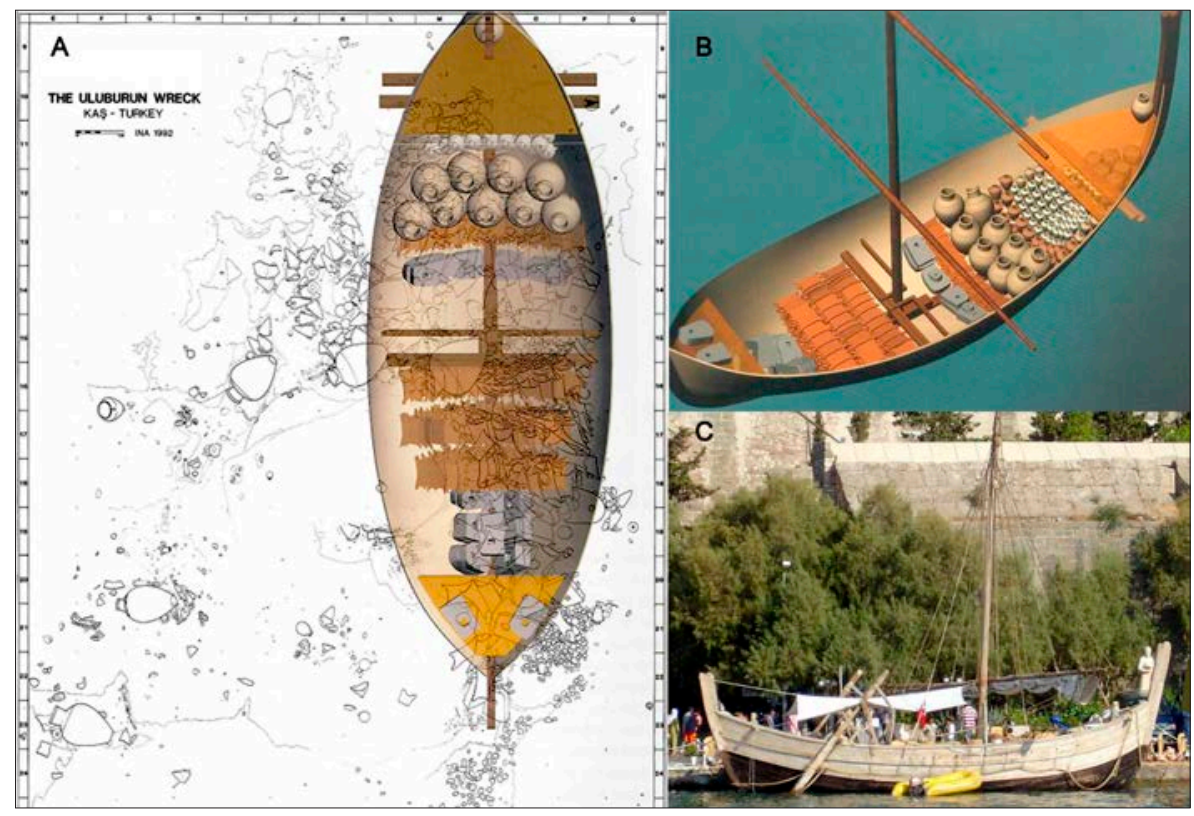

No obstante, muchos investigadores ya desde su publicación no aceptan el carácter prehistórico de estos grabados que los consideran modernos por su surco profundo en $\mathrm{V} y$ testimonios orales de ancianos que vieron trazar alguna figura (Peña Santos 1976: 149-152; Peña Santos y Vázquez Varela 1979: 100; García Alén y Peña Santos 1980: 79-80; Peña Santos y Rey García 2001: 30; Costas y Peña Santos 2005: 185 n. 1; 2011: 134-135 n. 2), e incluso el propio Alonso Romero (1979: 189 n. 3; 1994: 17) ha reconocido que algunos de los barcos pudieron ser vueltos a grabar de nuevo en época medieval. Aceptando la presencia de grabados más tardíos como los cruciformes, se acepta su identificación como barcos prehistóricos, concretamente mediterráneos (Ruiz-Gálvez 2005: 320).

A ello se sumó la posterior identificación de dos barcos pintados en el abrigo de arte esquemático de Laja Alta con barcos de junco o papiro (Alonso Romero 1993; 1994), artículos coetáneos con el descubrimiento de Auga dos Cebros.

En la interpretación del casco como un barco de junco o papiro, se plantea que las tres líneas verticales que se conservan y están separadas por intervalos regulares, de los que habrían 8 en total, 5 no conservadas, corresponden a las ataduras de los haces de junco o papiro, sujetando el estay de la proa y la burda de la popa la curvatura del casco, pues considera que en madera no podría soportar la tensión del estay y la burda que están amarrados a la roda y el codaste (Alonso Romero 1995: 139; 2011: 120).
El disco circular del remate de la proa o roda sería el disco solar y el remate del codaste de la popa sería una flor de loto a medio abrir (Alonso Romero 1995: 139; 2011: 121). En conclusión, propone que se trataría de una embarcación de origen o influencia egipcia, anterior al 2400 a.C. (Alonso Romero 1995: 140), esto es, de comienzos del Calcolítico Final, por lo que fecha el grabado gallego entre el 2400-2000 a.C. (Alonso Romero 2011: 122).

Sin embargo, la no continuidad de los trazos verticales al comenzar a elevarse la roda y el codaste en la proa y popa no necesariamente sugieren un barco de junco o papiro, pues las ataduras de los haces de junco deben estar en todo el recorrido de su casco (Mederos y Escribano 2008: 67). No obstante, desde una perspectiva egipcia como la defendida por Alonso, podría argumentarse que a veces tanto roda y codaste en los barcos de papiro son protegidos con fundas de piel que ocultaban los haces de junco (Landström 1961/1964: 18 fig. 28). Un remate en el aphaston o codaste en forma de flor de loto a veces aparece también en los hippoi fenicios (Mederos y Escribano 2008: 70 fig. 10).

Otro aspecto que a veces se olvida, pero puede apreciarse bien con la reconstrucción que se ha realizado del barco de Uluburun, es que los barcos en su parte baja estaban calafateados en negro para impermeabilizarlos. Esto da una forma más curva al barco en la parte no calafateada por el contraste del color marrón de la madera y el negro del calafateado, y más aún si se observaba el barco a distancia (fig. 6). 


\section{LA HIPÓTESIS DE UN BARCO INDÍGENA CON CASCO DE CUERO}

El yacimiento de Auga dos Cebros tiene su puerto más cercano en Oia, una pequeña ensenada arenosa rodeada de rocas, donde varan en la arena los barcos de fondo plano de los pescadores, denominados gamelas, que por su poco calado pueden acercarse a la costa y varar en la playa aprovechando la marea (Alonso Romero 1995: 137), lo que haría viable el uso de barcos de pesca con casco plano de cuero en la antigüedad.

En la mención de unas islas Estrímnidas en el Atlántico se indica que construyen siempre sus embarcaciones con pieles cosidas -aptant pellibus-, y muchas son las veces que recorren el vasto mar sobre el cuero (O.M. 105-107) (Villalba 1994: 54). Por otra parte, antes de la presencia de Decimo Junio Bruto en Galicia el 137 a.C., donde se enfrentó a los Brácaros y a los Galaicos, y celebró su victoria en Roma como cónsul el 136 a.C., se señala por Estrabón que usaban, hasta la época de Bruto, embarcaciones de cuero (Str. III, 3, 7).

Otro texto de Estrabón para la Galia indica que también El velamen era, en efecto, de cuero, para resistir la violencia de los vientos (...) Fabrican unos barcos de casco achatado y prominentes por la proa y por la popa, para aprovechar las mareas (Str. IV, 4, 1). También lo menciona Julio César (Bell. Gall. III, 13, 6) para la Galia en los barcos de una coalición de osimos, lexovios, mamnetes, ambiliatos, morinos, diablintes y menapios, pues usaban en lugar de velas, pieles y badanas delgadas (...) por juzgar que las velas no podrían resistir las enormes borrascas del Océano y la furia de los vientos. No obstante, estas velas de pieles, cosidas con las tripas de los animales, también se utilizaban en barcos con casco de madera.

Como sucede a veces en las embarcaciones escandinavas, los trazos verticales que están representados en el casco del barco de Auga dos Cebros suelen señalar las cuadernas en una embarcación de piel a contraluz (Landström 1961/1964: 53). Por esta razón hace unos años sugerimos que "quizás" el barco de Santa María de Oia podría ser un barco indígena con casco forrado de piel. Sin embargo, creemos que hay varios factores que no apoyan esta breve opinión preliminar que hicimos, en la cual nuestro objetivo principal era descartar que la embarcación fuese un barco de juncos (Mederos y Escribano 2008: 67) (fig. 7).

1) Los barcos de cuero exigen notables cuidados para ser duraderos, y precisan destinar un volumen importante de pieles tratadas si se pasa de una barca a un barco de mayor eslora. En este sentido, Alonso Romero (1976: 121) recuerda que en el curragh de Maelduin habían tenido que poner tres pieles superpuestas para hacerlo más resistente.

2) Al carecer de quilla, a medida que aumentan las dimensiones del barco, es cada vez más fácil que pueda volcar.

3) Este problema de la quilla se acrecienta si la embarcación de cuero tiene vela, pues como señala Alonso Romero (1976: 123) la posibilidad de volcar es aún más alta, aunque a veces se utilizan en los umiaks esquimales o en los curraghs de las islas de Aran con un pequeño mástil abatible cercano a la proa.

4) No es un barco adecuado para transportar mercancías pesadas pues carecen de quilla y son muy ligeras, en caso de no llevar una carga significativa. Este problema, en los curraghs de las islas de Aran (Alonso Romero 1976: 132), se salva llevando piedras y eliminándolas progresivamente mientras van pescando. Esto sería factible en caso de transportar mercancías muy valiosas como el estaño, el oro o el ámbar, pero no con otras como lingotes de cobre, bronces, sal, textiles, etc.

5) Finalmente, otro serio impedimento son las proyecciones verticales de la roda y codaste. Los barcos de cuero necesitan ser secados en la playa diariamente después de su uso, pues si no pierden su impermeabilidad, como sucede con los umiaks esquimales (Alonso Romero 1976: 132). El problema con un casco de cuero surgiría no sólo después de cada navegación, sino incluso en caso de lluvias torrenciales en invierno. Sin embargo, la primera embarcación de Auga dos Cebros, al tener unas proyecciones tan verticales de roda y codaste, como también apuntan Costas y Peña Santos (2005: 211 n. 48; 2011: 221 n. 4), no puede ponerse a secar virada al revés e impediría el secado diario del casco una vez usado.

\section{LA HIPÓTESIS DE UN BARCO CON CASCO DE MADERA DE LA EDAD DEL BRONCE}

Si descartamos las dos opciones de un casco de junco o de un casco de cuero, la opción más aceptable es que el barco representado cuente con un casco de madera. Sin embargo, hay distintas opiniones que influyen en la atribución cronológica que se propone para el barco. El elemento principal que apoya que se trata de un barco de madera es la presencia de una serie de trazos verticales en el casco, separados por intervalos regulares, de los que se 
Fig. 7: A: Grabados de barcos de Madsebakke, Bornholm (Dinamarca) con indicación de líneas verticales que señalan las cuadernas en una embarcación de piel a contraluz. Museo de Bornholm. B: Reconstrucción de un barco para unos 20 remeros con casco de cuero en el que se aprecian las cuadernas, a partir del barco de madera de Hjortspring, isla de Als (Dinamarca) del s. IV a.C., según Landstrom (1961/1964: 53).

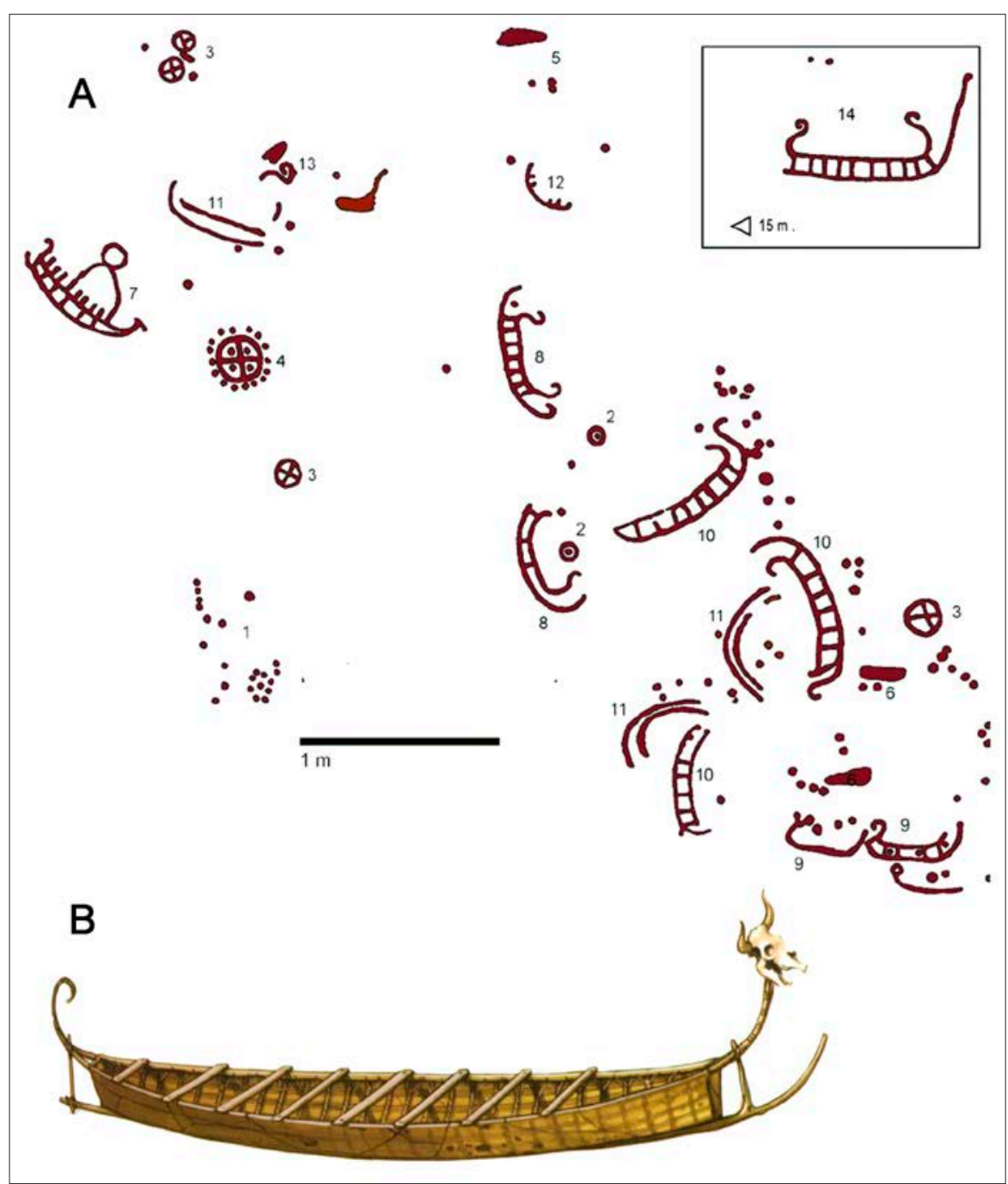

conservan tres, de unos ocho posibles, que nos podrían indicar la presencia de las aberturas regulares en el casco por la existencia de una bancada de ocho remeros por cada lado y 16 en total.

Una de las propuestas menciona que se trataría de un barco mediterráneo de la primera mitad del II milenio a.C., 2000-1500 a.C. (Costas y Peña Santos 2005: 213, 2006: 285 y 2011: 307). En una pequeña nota indican que el referente parecen ser los barcos minoicos, no se puede obviar la similitud (...) de los sellos cretenses: la vela desmontada, la indicación de mástil y cabos y las líneas verticales en el casco (Costas y Peña Santos 2011: 217 n. 1). La afinidad minoica vendría reforzada por la presencia de grabados con diseños de laberintos de modelo cretense entre los grabados gallegos (Costas y Peña Santos
2005: 211; 2011: 272). Sin embargo, los barcos minoicos, aunque usan vela y tienen remeros, siendo los mejores ejemplos por su detalle los de Akrotiri del Heládico Final IA (Wedde 2000: 321-322 no 613-619), ca. 1650-1480 a.C., no tienen bancadas de remeros bajo una cubierta. Este detalle es importante porque el barco de Auga dos Cebros no indica la existencia de una cubierta separada de los remeros (fig. 8).

Por otra parte, los autores se apoyan para su datación en la cronología que atribuyen a los grabados de los ciervos al galope, con un estilo propio de la zona SO de Galicia (Costas y Peña Santos 2002; 2005: 193; 2011: $101 \mathrm{n}$. 10, 102 n. 12), que asignan como muy reciente al Bronce Inicial, dentro de la cronología que proponen del grupo galaico de arte rupestre $c a$. 2500-1800 a.C. (Peña Santos 


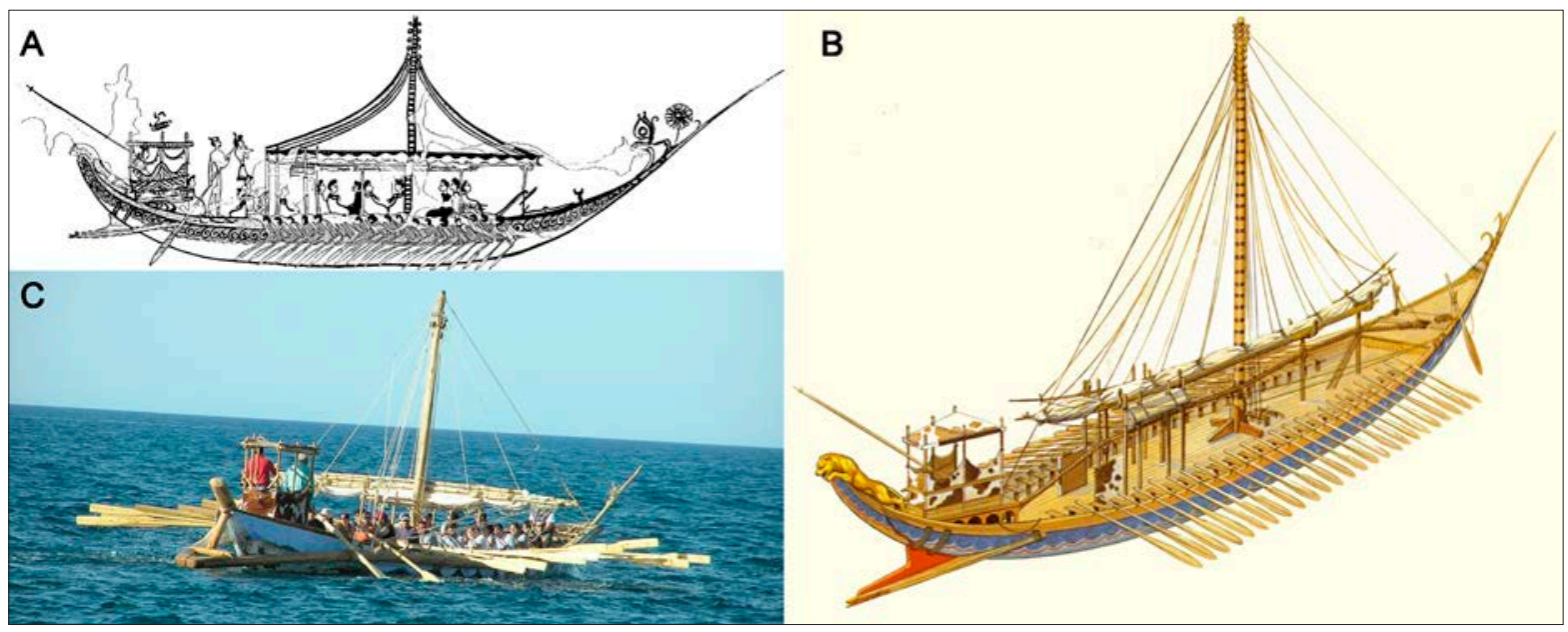

Fig. 8: A: Barco de remeros sin cubierta de Akrotiri, Minoico Final IA, ca. 1650-1480 a.C. (Wedde, 2000). B: Reconstrucción del barco de Akrotiri. C: Reconstrucción experimental del barco de Akrotiri.

2003: 76-92; Costas y Peña Santos 2005: 203; 2011: 272), aunque el ciervo principal está rebajado su superficie a modo de bajorrelieve, una técnica excepcional también presente en Coto das Sombriñas en Tourón (Pontecaldelas, Pontevedra).

Si resulta complejo admitir la presencia de un barco minoico en el Atlántico, al menos con los datos actualmente disponibles, no lo sería tanto que nos encontrásemos con un navío del tipo de Uluburun de ca. 1300 a.C. (Pulak 1998; 2008), donde su finalidad principal era su capacidad de carga. En esta opción de un barco 300 años más reciente, el aspecto más discutible es que el barco de Auga dos Cebros parece tener una bancada de remeros por la presencia de las líneas verticales en el casco, ausentes en Uluburun.

Una segunda opción sería un barco postmicénico de remeros del Heládico Final IIIB2 y IIIC entre fines del s. XIII y mediados del XII a.C., 1210-1150 a.C. (Ruiz-Gálvez 2005: 319; 2009: 47; 2013: 276, 278). Esta hipótesis es la que parece seguir González Ruibal (2004: 289; 2006-07: 137) quien fecha el barco a finales de la Edad del Bronce $y$ también con prudencia Mielke y Schuhmacher (2011: 78).

La tercera opinión es que se trataría de una embarcación fenicia del 900-800 a.C., cuando comienzan a frecuentar la costa portuguesa, que es la hipótesis de Guerrero (2009: 85-86), quien lo explicita en base a una visión en rayos $\mathrm{X}$ donde los trazos verticales en el casco responderían las cuadernas del interior del barco. Esta es la idea que parecen retomar Pereira y Arruda (2017: 197198) cuando lo fechan en el Hierro Inicial, a finales del s. VIII a.C., siguiendo rutas de cabotaje costero.
Desde nuestro punto de vista, se trata de un barco del Bronce Final como sugirió inicialmente Ruiz-Gálvez y trataremos de explicitarlo en detalle para enmarcar bien la banda cronológica de la embarcación, algo que no se ha desarrollado adecuadamente.

Por la documentación iconográfica que disponemos, hacia el Heládico Final IIIA2-IIIB (Wedde 1999: 465), ca. 1375-1185 AC, se produjo la aparición de barcos con mayor capacidad náutica y bancadas de remeros, de acuerdo con la tumba ${ }^{\circ} 3$ de Enkomi (Sjöqvist 1940: fig. 20/3), del Heládico Final IIIB, dentro del tipo V de galera micénica de Wedde (2000: 324-325 no 644-645), que muestra detalles muy interesantes como la presencia de una cubierta superficial, por encima de las bancadas de los remeros, donde se sitúan los guerreros portado espadas en el cinto. Por otra parte, tanto la proa como la popa son elevadas al proyectarse la roda y el codaste, presentando un akroterion con cabeza de ave (fig. 9).

El aumento del número remeros fue fundamental porque dio mayor maniobralidad a los barcos para entrar en combate o escapar de los piratas, facilitaba la entrada en los puertos, etc.

Este modelo de barco fue optimizándose a principios del s. XII a.C., incrementándose el número de remeros para acentuar la velocidad y capacidad de sorpresa si se realizaban saqueos en tierra, utilizando brioles para un mejor despliegue y recogida de la vela, que así no molestaba a los combatientes en la cubierta y fue más efectiva en los trayectos con el viento en contra (Robert 1991: 59, lám. 17-20; Emanuel 2014: 25-26). El detalle de las velas 
Fig. 9: A: Crátera del Heládico Final IIIB de la tumba 3 de Enkomi (Chipre), Medelhavsmuseet, Estocolmo, E 3262. B: Detalle del barco de Enkomi con representación esquemática de remeros y una cubierta superior con guerreros armados con espadas. C: Dibujo de los dos barcos representados, con proa y popa elevadas, y akroterion con cabeza de ave (Sjöqvist 1940: fig. 20/3).
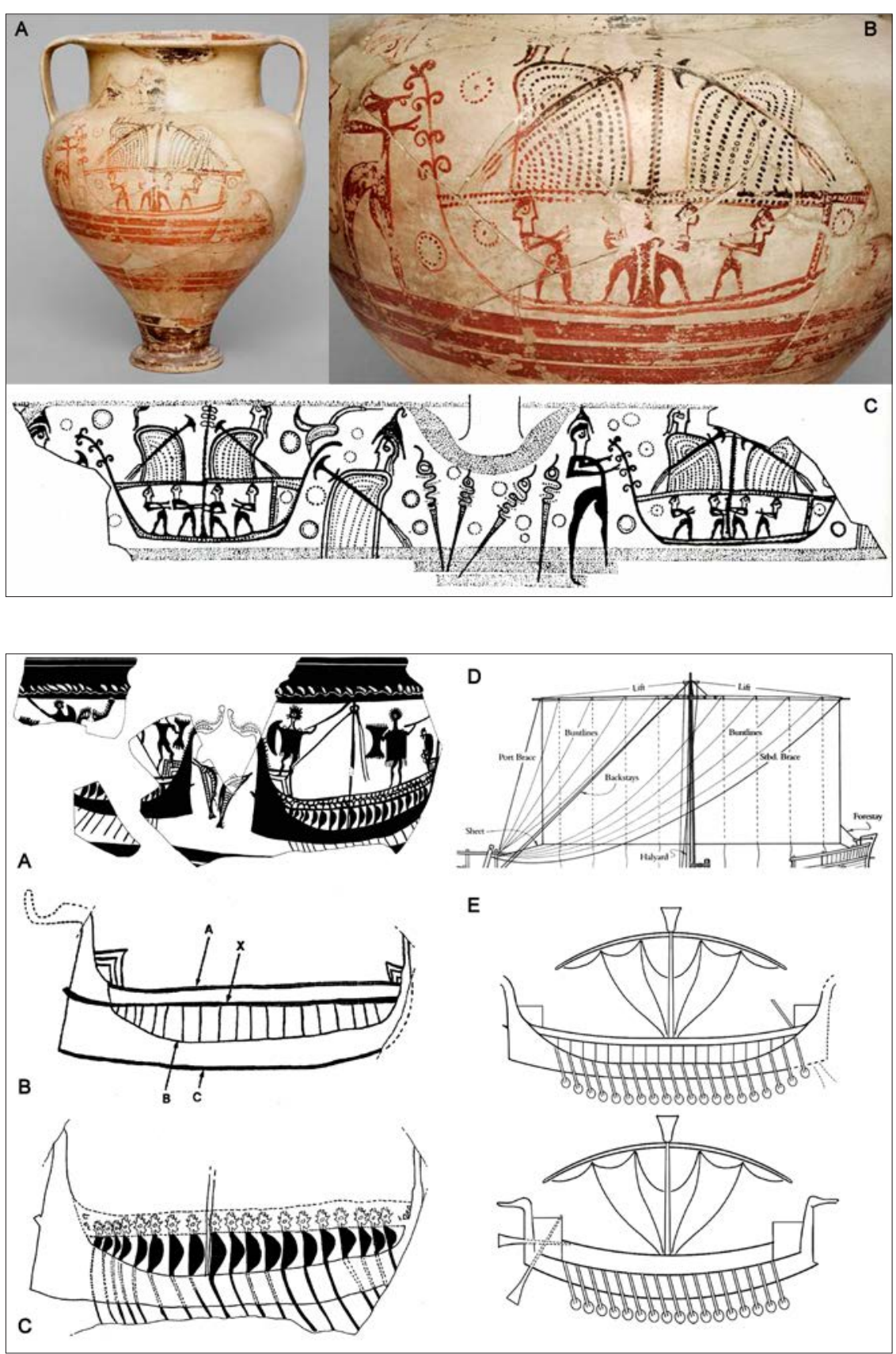

Fig. 10: A: Dibujo de dos barcos enfrentados con guerreros en la cubierta en una crátera del Heládico Final IIIC Medio, 1130-1070 a.C. de Pyrgos Livanaton, la ciudad de Kynos-Cino en el catálogo de las naves (Mountjoy 2011: 485 fig. 2), Museo de Lamia. B: Detalle del casco donde se observa la bancada de los remeros señalada con líneas verticales y una cubierta superior (Wachsmann 1997: 347 fig. 13) C: Reconstrucción de los remeros por debajo de la cubierta superior (Wachsmann 1997: 349 fig. 16). D: Vela con brioles -butlines- para su mejor despliegue y recogida. E: Velas recogidas formando semicirculos en el barco de Pyrgos Livanaton, tal como están representadas en el templo mortuorio de Ramsés III en Medinet Habu (Luxor) en la batalla naval de Ramsés III contra los Pueblos del Mar en el Delta del Nilo $c a$. 1176 a.C. (Emanuel 2014: 33 fig. 7). 


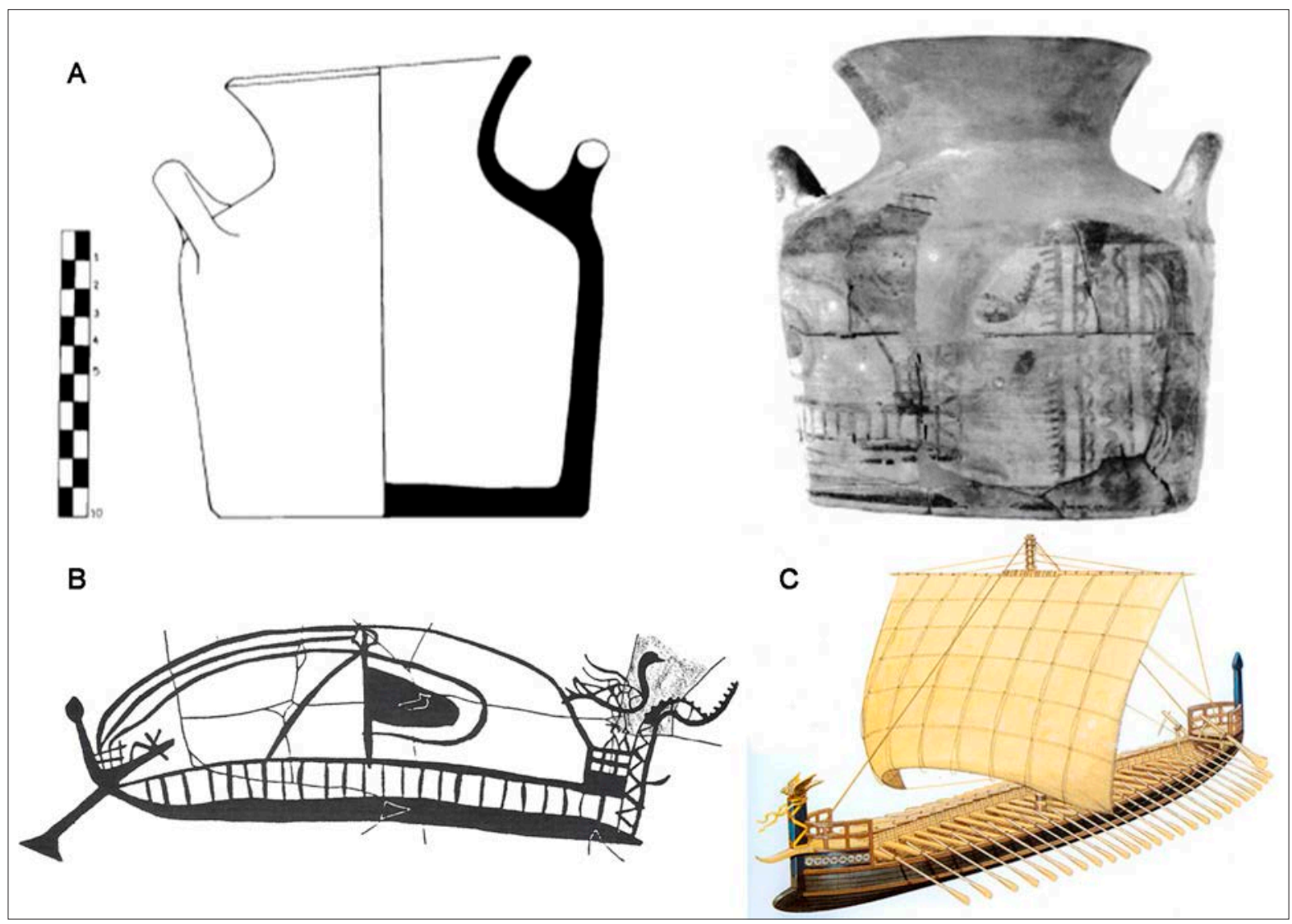

Fig. 11: A: Pyxis del Heládico Final IIIC Medio, 1130-1070 a.C., de la tumba 11 de Tragana (Korrés 1989: 202 fig. 3), Museo Nacional de Atenas, 6098. B: Detalle del barco con tajamar en la proa y 24 trazos verticales que indican 50 remeros de un pentecóntero (Wedde 1999: lám. 89/B7), como los que participaron en la Guerra de Troya, ca. 1196-1186 a.C. C: Reconstrucción del barco de Tragana.

superficial por encima de la cubierta de las cabezas de los remeros, y además los dos cables que caen colgando del mástil sugieren que son brioles para recoger la vela (Wachsmann 1997: 349 fig. 16; Emanuel 2014: 28). Por otra parte, la escena naval, que ha sido reconstruida por Mountjoy (2011: 485 fig. 2), refleja el combate entre dos galeras con los guerreros sobre la cubierta, armados de escudo circular y lanza, sin utilizar arcos y flechas (fig. 10).

Aún no se trata de una galera del tipo VI de Wedde (1999: 467, 469), que son las primeras que presentan la proyección del tajamar desde la quilla, el cual facilita la navegación cortando las olas, actuaba como contrapeso hundido del barco y ayuda a entrar el barco en las playas de arena para propiciar un rápido desembarco de los guerreros. Sin embargo, el tajamar no debe confundirse con un espolón en el barco de uso militar, que no aparece hasta $c a .850$ a.C. (Wedde 2000: 172).
Uno de los mejores ejemplos del nuevo tipo de galeras más largas, con más remeros y menos peso, es el pyxis del Heládico Final IIIC Medio de la tumba 11 en tholos de Tragana (Korrés 1989: 200 fig. 1; Wachsmann 1997: 346-347 fig. 11; Wedde 2000: $324 \mathrm{n}^{\mathrm{o}}$ 642), 1130-1070 a.C., donde aparte del tajamar en la proa, se observa proa y popa elevadas al proyectarse la roda y el codaste y los trazos verticales en el casco, 24, que conforman 25 espacios a cada lado, e indicarían la presencia de bancadas que suman 50 remeros (fig. 11).

Otro buen referente es el barco pintado en un larnax de arcilla de la tumba de Skapidara, cerca en Gazi, en el área de Knossos, del Minoico Final IIIB (Alexiou 1970: 253254 y 1972: 90-98; Wachsmann 1981: 203 fig. 18 y 1997: 346 fig. 10; Wedde 2000: 320 no 608), 1340-1190 a.C., en el cual el tajamar es muy evidente, presenta un prótomo de animal, ave o caballo, 26 líneas verticales paralelas en el casco indicativas de los remeros, también probablemente 
50 remeros, proa y popa levantada con doble estay y doble burda, representación de la verga con la vela recogida y un toldo de una caseta para resguardarse. Esta embarcación, si prescindimos del tajamar, es claramente el modelo más próximo al primer barco de Auga dos Cebros, y se podría argumentar incluso que hay un posible tajamar en la segunda embarcación de Auga dos Cebros (fig. 12).

\section{CONCLUSIONES}

El primer barco de Auga dos Cebros parece corresponderse con una embarcación con casco de madera, con unos 16 remeros, que cuenta con un mástil en posición central, aunque no representa la vela, con proa y popa elevadas al proyectarse la roda y el codaste. Una de las opciones es que al no indicarse una cubierta superficial es que se trate de un barco de carga del tipo de Uluburun, ca. 1300 a.C., sin embargo, parece presentar una bancada de remeros por las líneas verticales en el casco. Este dato apunta más a que se trate de un barco contemporáneo ya con cubierta superficial, los cuales comienzan con seguridad a partir del Heládico Final IIIB, 1325-1185 a.C., o Chipriota Final IIC, 1300-1190 a.C., de acuerdo con su representación en la tumba $n^{\circ} 3$ de Enkomi. Estas embarcaciones tienen mayor capacidad de maniobra en los puertos, para combatir o escapar de los piratas. Durante el s. XIII, o muy a inicios del s. XII a.C., antes de la crisis de los Pueblos del Mar, ya hubo una importante innovación, que tampoco podemos atribuir con seguridad al barco de Auga dos Cebros, pero que podría ser importante al facilitar la navegación de ascenso hacia Galicia, el uso de brioles para un mejor despliegue y recogida de la vela, que hizo la navegación más efectiva en los trayectos con el viento en contra, sólo constatado en iconografía durante el segundo enfrentamiento de Ramsés III contra los Pueblos del Mar en el Delta del Nilo el 1176 a.C.

La otra progresiva mejora fue el incremento del número de remeros, aunque el número máximo de remeros que sugiere el grabado de Auga dos Cebros sólo es ocho por banda, 16 en total.Sin embargo, no cabe descartar que hayan sido más, pero se simplifican en el grabado. La representación náutica que presenta más similitudes con Auga dos Cebros es el barco del larnax de la tumba de Skapidara, cerca en Gazi, del Minoico Final IIIB, 1340-1190 a.C., el cual nos advierte que el aumento del número de remeros ya se había producido desde el s. XIII a.C. pues se trata de una pentecóntera de 50 remeros, 25 por banda.
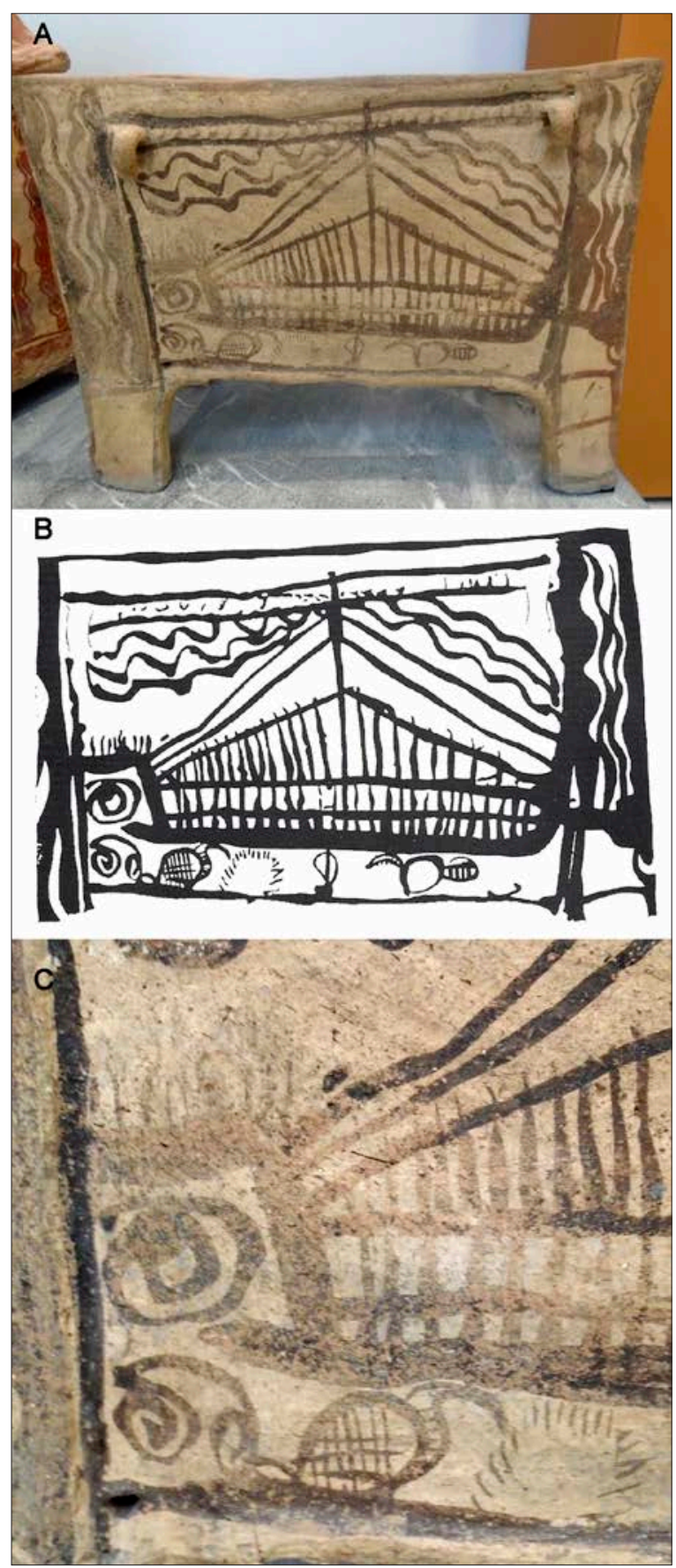

Fig. 12: A: Larnax de la tumba de Skapidara, cerca en Gazi, del Minoico Inicial IIIB, 1340-1190 a.C., Museo Arqueológico de Heraklion, 18.985. B: Detalle del barco con 26 líneas verticales paralelas en el casco indicativas de un pentecóntero de 50 remeros, mástil con verga y vela recogida y el toldo de una caseta para resguardarse (Wachsmann 1997: 346 fig. 10). C: Detalle del tajamar y del akroteion con prótomo de animal. 


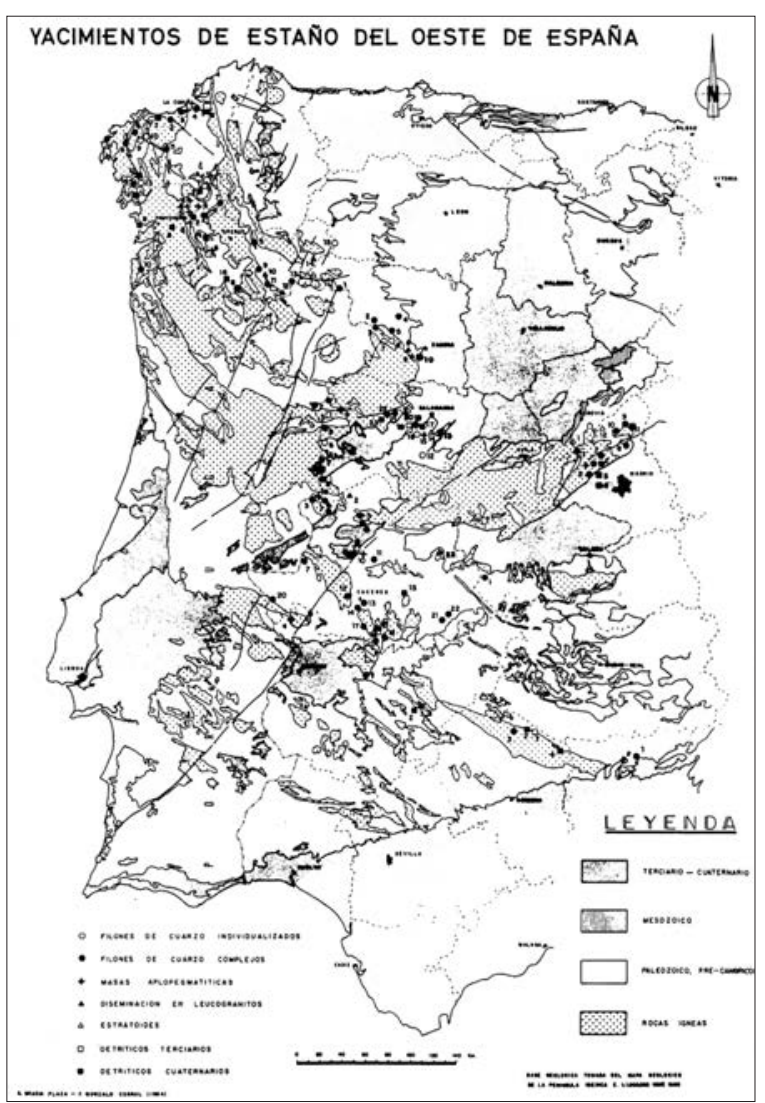

Fig. 13: Yacimientos de estaño en la fachada occidental de la península Ibérica (Gonzalo Corral y Gracia 1985: 280 fig.).

La cronología de un barco como el de Auga dos Cebros no debería sobrepasar, a priori, el inicio del Bronce Final IIIA en la Península Ibérica o el Protogeométrico en el Egeo, a partir del 1050 a.C., cuando ya son habituales galeras con numerosos remeros y un tajamar desarrollado (Wedde 1999: 471, lám. 92), lo que nos situaría entre el s. XII y primera mitad del s. XI a.C.

La segunda cuestión a valorar es si se trata de un barco foráneo o local. Aunque las innovaciones náuticas se difunden rápidamente, una representación de un barco levantino o egeo no debe sorprendernos pues sabemos que el estaño atlántico estuvo siendo suministrado desde la fachada atlántica de la Península Ibérica hacia el Mediterráneo. Sin embargo, tampoco cabe descartar que se tratase de un barco del S de la península Ibérica procedente de la región gaditana, de la desembocadura del Guadalquivir o de la confluencia de los ríos Tinto y Odiel, áreas costeras especializadas en el comercio con el Atlántico (fig. 13).
La zona más accesible desde el mar y principal fuente de obtención de estaño filoniano y aluvial en la península Ibérica se encontraba en Galicia, con prolongaciones por Tras-os-Montes, las Beiras, Salamanca y Cáceres, destacando las cuencas mineras de Noya (La Coruña), Lalín (Pontevedra), Carballo (Orense), Verín (Orense), La Gudiña (Orense) y Viana del Bollo (Orense) (Obermaier 1923: 37-39, fig. 3; Monteagudo 1950: 2; Guzmán 1983: 46-49; Gonzalo Corral y Gracia 1985: 280 fig., 281-283; Merideth 1998; Comendador et al. 2014).

Otra cuestión que debe tenerse en cuenta es que desde finales del s. XII a.C. las fuentes mencionan la fundación del santuario de Melqart en Gadir, ca. 1106 a.C., 80 años después de la caída de Troya, según recoge Veleyo Patérculo (Hist. Rom. I, 2, 3). Incluso algún autor como Mela retrotrae su antigüedad, el templo de Hércules Egipcio (...) Los tirios lo construyeron (...) desde la época de Troya (Mel. III, 6, 46), ca. 1186 a.C. según los cálculos de Timeo en el s. IV-III a.C. (Mederos 2007: 97-98 tabla 1). No obstante, la presencia fenicia no está confirmada arqueológicamente hasta mediados del s. X a.C., 975-960 a.C., con una frecuentación previa más intensa desde el 1015 a.C. (Mederos 2006: 172) o bien el 940 a.C. (González de Canales et al. 2009: 3), retrotrayendo la propuesta inicial del 900-770 a.C. (González de Canales et al. 2006: 115), o bien poco después, 930-830 a.C. (Nijboer y van der Plicht 2006: 31), aunque otros investigadores como Núñez (e.p.: 145-146) lo sitúan entre el 850775 a.C. En todo caso, las menciones de la fundación de un santuario que facilitaría el comercio dentro de un espacio sacro para asegurar las transacciones nos están indicando que debió comenzar una frecuentación por marinos de la ciudades del Levante de las costas del S de la península Ibérica, y bien estos mismos barcos, u otros procedentes del S de Andalucía debieron comenzar a visitar más regularmente el litoral atlántico para obtener oro y en particular estaño aluvial, la única alternativa marítima cuando existían problemas de suministro y desabastecimiento del estaño que llegaba por rutas terrestres procedente de Afganistán, Irán, Tajikistan o Uzbekistan.

La fecha de ca. 1106 a.C. para la fundación del santuario en Gadir podría vincularse con las campañas de Tiglath-pileser I, Tukultī-apil-Ešarra, 1113-1076 a.C. (Jeffers 2013: 188 tabla 2), cuyas expediciones llegaron hasta Arwad, en el Mediterráneo, situando a toda la cuenca siria y libanesa del río Orontes bajo su área de influencia, y exigiendo a pagar tributo a los puertos fenicios de Arwad, Gubal-Biblos y Sidón (Pritchard 1955; ANET 
274-275). Arwad pasó probablemente a ser dependencia asiria pues después de conquistar Amurru y recibir el tributo de todos los reyes locales, Tiglath-pileser I navegó desde Arwad hasta Samuru, visitó Biblos y Sidón, y cazó un animal marino que denomina nahiru o caballo del mar (Grayson 1991: RIMA II, A.0.87.3, 16-25; Yamada 2005: 33-34, 52 tabla 3). Como la campaña de Tiglath-pileser I fue poco después de su décimo año de reinado correspondería a ca. 1104 a.C.

Por todo ello, creemos viable situar el barco de Auga dos Cebros entre el 1200-1050 a.C., en un momento en que la notable presencia de cuchillos de hierro, ponderales o ámbar báltico durante el Bronce Final IIC (Vilaça 2006; 2012; Mederos 2009) sugieren la presencia de barcos que navegaban y comerciaban regularmente por las costas atlánticas de la península Ibérica.

\section{NOTA}

1. A la memoria de Víctor Guerrero Ayuso.

\section{AGRADECIMIENTOS}

Queremos agradecer a José Luis López Castro atender a nuestras consultas. Este trabajo se adscribe al proyecto "La Ruta de las Estrímnides. Comercio Mediterráneo e Interculturalidad en el Noroeste de Iberia", HAR2015-68310-P, bajo la dirección de E. Ferrer Albelda, del Ministerio de Economía y Competitividad.

\section{BIBLIOGRAFÍA}

ALMAGRO GORBEA, M. (1995): La navegación prehistórica y el mundo atlántico, Guerra, exploraciones y navegación: del Mundo Antiguo a la Edad Moderna (El Ferrol 1994), Universidad Menéndez y Pelayo, Betanzos, 13-35.

ALEXIOU, S. (1970): Mikrai Anaskaphai kai Perisulloge Arxaion eis Kreten, Praktika tes en Athenais Arkhaiologikes Etaireias 1970, 252-255.

ALEXIOU, S. (1972): Larnakes kai Aggeia ek Taphou Para to Gazi, Irakliou, Archaiologiki Ephemeris 1972, 86-98.

ALONSO ROMERO, F. (1976): Relaciones atlánticas prehistóricas entre Galicia y las islas Británicas y medios de navegación, Vigo.

ALONSO ROMERO, F. (1993): Los testimonios más antiguos de los medios de navegación entre el Mediterráneo y el Atlántico: las embarcaciones de juncos en el arte rupestre de la Península Ibérica, Mediterráneo 2, 265-284.
ALONSO ROMERO, F. (1994): Prehistoric Boats in the RockPainting of Cádiz and in the Rock-Carving of Northwestern Spain, Crossroads in Ancient Shipbuilding. 6th International Symposium on Boat and Ship Archaeology (Roskilde 1991) (C. Westergahl, ed.), Oxbow Monographs 40, Oxford, 11-20.

ALONSO ROMERO, F. (1995): La embarcación del petroglifo Laxe Auge dos Cebros (Pedornes, Santa María de Oia, Pontevedra), XXII Congreso Nacional de Arqueología (Vigo 1993), Vigo, 137-145.

ALONSO ROMERO, F. (2011): La embarcaciones prerromanas del área atlántica europea, Anuario Brigantino 34, 93-158.

BRADLEY, R.; FÁBREGAS, R. (1996): Petroglifos gallegos y arte esquemático: una propuesta de trabajo, Homenaje al Profesor Manuel Fernández-Miranda (M. A. Querol, T. Chapa, eds.), Complutum. Extra 6 (1), Madrid, 103-110.

CÉSAR, G. JULIO (1996): Guerra de las Galias, trad. V. García Yebra y H. Escolar Sobrino, Biblioteca Clásica Gredos 50, Gredos, Madrid.

COLES, J. (2003): And on they went...processions in Scandinavian Bronze Age rock carvings, Acta Archaeologica 74, 211-250.

COMENDADOR, B.; FIGUEIREDO, E.; FONTE4S, J.; MEUNIER, E. (2014): La primera minería y metalurgia del estano en la Península Ibérica: aportaciones al estado de la cuestión, XV Congreso Internacional sobre Patrimonio Geológico y Minero (Logrosán 2014), Logrosán, 21-40.

COSTAS GOBERnA, F. J.; NOVOA, P.; SANROMÁN, J. A. (1995): Santa María de Oia. Sus grabados rupestres, XXII Congreso Nacional de Arqueología (Vigo 1993) II, Vigo, 131-135.

COSTAS GOBERNA, F. J.; PEÑA SANTOS, A. DE LA (2002): A fauna e o ser humano na prehistoria do Baixo Miño a través dos gravados rupestres, Monografías da Asociación Naturalista 'Baixo Miño' 13, A Guarda.

COSTAS GOBERNA, F. J.; PEÑA SANTOS, A. DE LA (2005): Oia. Arte rupestre y embarcaciones en la prehistoria de Galicia, Glaucopis, Boletín del Instituto de Estudios Vigueses 11, 185-223.

COSTAS GOBERnA, F. J.; PEÑA SANTOS, A. DE LA (2006): Los barcos de los Petroglifos de Oia. Los tesoros del hechicero y una nueva embarcación, Glaucopis, Boletín del Instituto de Estudios Vigueses 12, 277-292.

DAKORONIA, P. (1989): War-Ships on Sherds of LHIIIC Kraters from Kynos, Tropis II. 2nd International Symposium on Ship Construction in Antiquity (Delphi 1987) (H. Tzalas ed.), Delphi, 117-122.

EMANUEL, J. P. (2014): Sea Peoples, Egypt, and the Aegean: the transference of maritime technology in the Late BronzeEarly Iron transition (LH IIIB-C), Aegean Studies 1, 21-56.

ESTRABÓN (1992): Geografía. Libros III-IV, trad. de M M $^{\mathrm{a}}$.J. Meana y F. Piñero, Biblioteca Clásica Gredos 169, Gredos, Madrid. 
FÁBREGAS, R.; RUIZ-GÁLVEZ, M. L. (1995): El Noroeste de la Península Ibérica en el $\mathrm{III}^{\mathrm{er}}$ y $\mathrm{II}^{\circ}$ Milenios: Propuestas para una síntesis, Sagvntvm-PLAV 30, 191-216.

GARCÍA ALÉN, A.; PEÑA SANTOS, A. DE LA (1980): Grabados rupestres de la provincia de Pontevedra, Fundación 'Pedro Barrié de la Maza. Conde de Fenosa', La Coruña.

GONZÁLEZ DE CANALES, F.; SERRANO, L.; LLOMPART, J. (2006): Las evidencias más antiguas de la presencia fenicia en el sur de la península, Tiempos de Púrpura. Málaga antigua y antigüedades hispanas (Vélez-Málaga 2006), I (M. Corrales et al., eds.), Málaga, 105-128.

GONZÁLEZ DE CANALES, F.; SERRANO, L.; LLOMPART, J. (2009): The two phases of Western Phoenician expansion beyond the Huelva finds: An interpretation, Ancient West and East 8, 1-20.

GONZÁLEZ RUIBAL, A. (2004): Facing two seas: Mediterranean and Atlantic contacts in the North-West of Iberia in the First Millennium B.C., Oxford Journal of Archaeology 23 (3), 287-317.

DOI: https://doi.org/10.1111/j.1468-0092.2004.00213.x

GONZÁlEZ RUIBAL, A. (2006-07): Galaicos. Poder y comunidad en el Noroeste de la Península Ibérica (1200 a.C.-50 d.C.), I-II, Brigantium 18. A Coruña.

GONZALO CORRAL, F. J.; GRACIA PLAZA, A. S. (1985): Yacimientos de estaño del Oeste de España: ensayo de clasificación y caracterización económica, Revista de Xeoloxia Galega e do Hercínico Peninsular 9, 256-303.

GRAYSON, A. K. (1991): Assyrian Rulers of the Early First Millennium BC I (1114-859), The Royal Inscriptions of Mesopotamia, Assyrian Periods 2, Toronto.

GUERRERO AYUSO, V. (2009): Las Naves del Kerné. II. Navegando por el Atlántico durante la protohistoria y la antigüedad, Los Fenicios y el Atlántico. IV Coloquio Internacional del Centro de Estudios Fenicios y Púnicos (Tenerife 2004) (R. González Antón, F. López Pardo, V. Peña Romo, eds.), Centro de Estudios Fenicios y Púnicos, Madrid, 69-142.

GUZMÁN, F. V. (1983): Depósitos minerales de España, Instituto Geológico y Minero de España, Madrid.

JALHAY, E. (1926): Los grabados rupestres del extremo Sudoeste de Galicia. Alrededores de Oia. Provincia de Pontevedra, Boletín de la Comisión Provincial de Monumentos de Orense 7 (167), 373-385.

JALHAY, E. (1927-29): Algunos ejemplares del arte rupestre en los alrededores de Oia. Pontevedra, Boletín de la Comisión Provincial de Monumentos de Orense 8 (184), 305-312.

JALHAY, E. (1931): Nuevas manifestaciones de arte rupestre del Noroeste de la Península Ibérica, Boletín de la Comisión Provincial de Monumentos de Orense 9 (199), 225-235.

JALHAY, E. (1932): Novas descubertas prehistóricas no Sudoeste de Galicia e Norte de Portugal, XIII Congreso de la Asociación Española para el Progreso de las Ciencias-VI Congresso de la Associaçao Portuguesa para o Progresso das Sciências (Lisboa 1932), Madrid.
JEFFERS, J. A. (2013): Tiglath-Pileser I: a light in a 'Dark Age', $\mathrm{PhD}$ dissertation, University of Pennsylvania, Philadelphia.

KORRÉS, G. S. (1989): Representation of a Late Mycenaean Ship on the Pyxis from Tragana, Pylos, Tropis I. 1st International Symposium on Ship Construction in Antiquity (Piraeus 1985) (H. Tzalas, ed.), Delphi, 177-202.

LANDSTRÖM, B. (1961): Skeppet, Bokförlaget Forum AB, Stockholm.

LANDSTRÖM, B. (1961/1964): El buque, Editorial Juventud, Barcelona.

MACIÑEIRA Y PRADO, F. (1947): Bares. Puerto hispánico de la primitiva navegación occidental (F. Bouza-Brey ed.), Instituto Padre Sarmiento de Estudios Gallegos-Consejo Superior de Investigaciones Científicas, Santiago de Compostela.

MARK, S. E. (2000): Homeric Seafaring, PhD dissertation, Texas A\&M University, College Station, TX.

MEDEROS MARTÍN, A. (2006): Fenicios en Huelva, en el siglo X AC, durante el reinado de Hîram I de Tiro, Spal 15, 167-188. DOI: https://doi.org/10.12795/spal.2006.i15.08

MEDEROS MARTÍN, A. (2007): La crisis del siglo XII a.C. Pueblos del Mar y Guerra de Troya ca. 1215-1175 a.C., Spal 16, 93-153.

DOI: https://doi.org/10.12795/spal.2007.i16.06

MEDEROS MARTÍN, A. (2009): Metal para los dioses. La secuencia del grupo Baiões durante el Bronce Final II y el comercio chipriota de hierro hacia Portugal (1200-1050 AC), Estudios de Prehistoria y Arqueología en Homenaje a Pilar Acosta Martínez. (M. R. Cruz Auñón y E. Ferrer eds.), Universidad de Sevilla, Sevilla, 279-304.

MEDEROS, A.; ESCRIBANO, G. (2008): Caballos de Poseidón. Barcos de junco y hippoi en el Sur de la Península Ibérica y el litoral atlántico africano, Sagvntvm-PLAV 40, 63-78.

MELA, P. (1989): Corografía, trad. C. Guzmán Arias, Universidad de Murcia, Murcia.

MERIDETH, C. (1998): An Archaeometallurgical Survey for Ancient Tin Mines and Smelting Sites in Spain and Portugal, Mid-Central Western Iberian Geographical Region, 19901996, B.A.R.-I.s. 714, Oxford.

MIELKE, D. P.; SCHUHMACHER, T. X. (2011): Zeugnisse prähistorischer Seefahrt? Die Felsbilder mit Schiffsdarstellungen von der Iberischen Halbinsel, Skyllis 11 (2), 74-87.

MONTEAGUDO GARCÍA, L. (1950): Casitérides, Emérita 18 (1), 1-17.

MOUNTJOY, P. A. (2011): A Bronze Age Ship from Ashkelon with Particular Reference to the Bronze Age Ship from Bademgedigi Tepe, American Journal of Archaeology 115, 483-488.

DOI: https://doi.org/10.3764/aja.115.3.0483

NELSON, H. H. (1930): Medinet Habu Reports. I. Earlier Historical Records of Ramses III: Plates 1-54, Oriental Institute Publications 10, The University of Chicago Press, Chicago, Ill. 
Auga dos Cebros (Pontevedra, Galicia): un barco del Bronce Final II en la fachada atlántica DE LA PENÍNSUla IBÉRICA (1325-1050 A.C.)

NIJBOER, A. J.; VAN DER PLICHT, J. (2006): An interpretation of the radiocarbon determinations of the oldest indigenousPhoenician stratum thus far, excavated at Huelva, Tartessos (south-west Spain), BABesch 81: 31-36. DOI: https://doi.org/10.2143/BAB.81.0.2014423

NOVOA ÁLVAREZ, P. (1995): Nuevos aportes del arte rupestre gallego, Revista de Arqueología 168, 6-15.

NÚÑEZ CALVO, F. (e.p.): Una lectura tipológica-secuencial de los materiales cerámicos orientales más antiguos encontrados en Huelva. I Fenici in Andalusia. Nuove acquisizione (M. Botto ed.), Collezioni di Studi Fenici, Roma, 79-154.

OBERMAIER, H. (1923): Impresiones de un viaje prehistórico por Galicia. II, Boletín Arqueológico de la Comisión Provincial de Monumentos Históricos y Artísticos de Orense 7 (149), 25-47.

PEÑA SANTOS, A. DE LA (1976): Antropomorfos en el arte rupestre prehistórico gallego, El Museo de Pontevedra 30: 141-175.

PEÑA SANTOS, A. DE LA (2003): Galicia. Prehistoria, castrexo e primeira romanización, A Nosa Terra, Vigo.

PEÑA SANTOS, A. DE LA; REY GARCÍA, J. M. (1979): Petroglifos de Galicia, Vía Láctea Historia, Oleiros, A Coruña.

PEÑA SANTOS, A. DE LA; VÁZQUEZ VARELA, J. M. (1979): Los petroglifos gallegos. Grabados rupestres prehistóricos al aire libre en Galicia, Cuadernos del Seminario de Estudios Cerámicos de Sargadelos 30, Sada, La Coruña.

PEREIRA, G. R.; ARRUDA, A. M. (2017): Boats carved on the Atlantic coast of the Iberian peninsula. Landscape, symbols and people, Recorded Places. Experienced Places. The Holocene rock art of the Iberian Atlantic north-west (A.M.S. Betthencourt, M. Santos, H.A. Sampaio y D. Cardoso eds.), B.A.R.-I.s. 2878, Oxford, 193-207.

PRITCHARD, J. B. (ed.) (1955): Ancient Near Eastern Texts Relating to the Old Testament (ANET), Princeton University Press, Princeton.

PULAK, C. (1998): The Uluburum shipwreck: an overview, The International Journal of Nautical Archaeology 27 (3): 188-224. DOI: https://doi.org/10.1111/j.1095-9270.1998.tb00803.x

PULAK, C. (2008): The Uluburun Shipwreck and Late Bronze Age Trade, Beyond Babylon: Art, Trade, and Diplomacy in the Second Millennium B.C. (J. Aruz, K. Benzel, J.M. Evans eds.), The Metropolitan Museum of Art Exhibition Catalog, New York, 299-305, artifact catalog: 306-310, 313-321, 324-333, 336-342, 345-348, 350-358, 366-378, 382-385.

REY DA SILVA, A. (2009): Iconografía Náutica de la Península Ibérica en la Protohistoria, B.A.R.-I.s. 1982, Oxford.

REY DA SILVA, A. (2015): Nautical Iconography from the Iberian Peninsula in Prehistory, Maritime Contacts of the Past: Deciphering Connections amongst Communities (S. Tripati ed.), Marine Archaeology Centre, CSIR-National Institute of Oceanography, Goa, 368-401.

RIUDAVETS Y TUDURY, P. (1867): Derrotero de las costas de España y Portugal desde el Cabo Trafalgar hasta el puerto de La Coruña, Dirección de Hidrografía, Madrid.
ROBERTS, O. T. P. (1991): The Development of the Brail into a Viable Sail Control for Aegean Boats of the Bronze Age, Thalassa: L'Égée Préhistorique et la Mer. 3rd Rencontre égéenne internationale (Corsica 1990) (R. Laffineur, L. Basch, ed.), Liège, 55-64.

RUIZ-GÁLVEZ PRIEGO, M. L. (2005): Representaciones de barcos en el arte rupestre: piratas y comerciantes en el tránsito de la Edad del Bronce a la Edad del Hierro, Mayurqa 30 (1), 309-339.

RUIZ-GÁLVEZ PRIEGO, M. L. (2009): San Brandanes de la Prehistoria. Navegación atlántica prefenicia, Los Fenicios y el Atlántico. IV Coloquio Internacional del Centro de Estudios Fenicios y Púnicos (Tenerife 2004) (R. González Antón, F. López Pardo, V. Peña Romo, eds.), Madrid, 39-50.

RUIZ-GÁLVEZ PRIEGO, M. L. (2013): Con el fenicio en los talones. Los inicios de la Edad del Hierro en la cuenca del Mediterráneo, Barcelona.

SJÖQVIST, E. (1940): Problems of the Late Cypriote Bronze Age, The Swedish Cyprus Expedition, Stockholm.

VELEYO PATÉRCULO, M. (2001): Historia Romana, trad. M. A. Sánchez Manzano, Biblioteca Clásica Gredos 284, Madrid.

VILAÇA, R. (2006): Artefactos de ferro em contextos do Bronze Final do territorio português: Novos contributos e reavaliação dos dados. Complutum 17, 81-101.

VILAÇA, R. (2012): Ponderais do Bronze Final-Ferro inicial do ocidente peninsular: novos dados e questões em aberto, Trueque, dinero y moneda en el Mediterráneo Antiguo (ss. $X-I$ a.C.). IV encuentro peninsular de numismática Antigua (Madrid, 2010) (M. P. García-Bellido, L. Callegarin y A. Jiménez, eds.), Anejos de Archivo Español de Arqueología 58, Madrid, 139-167.

VILLALBA I VARNEDA, P. (1994): Ora Marítima, Avieno. Ora Maritima. Descriptio Orbis Terrae. Phaenomena (J. Mangas, D. Plácido, eds.), Madrid, 31-169.

WACHSMANN, S. (1981): The ships of the Sea Peoples, The International Journal of Nautical Archaeology and Underwater Exploration 10 (3), 187-220.

DOI: https://doi.org/10.1111/j.1095-9270.1981.tb00030.x

WACHSMANN, S. (1997): Were the Sea Peoples Mycenaeans? The Evidence of Ship Iconography, Res Maritimae: $C y$ prus and the Eastern Mediterranean from Prehistory to Late Antiquity (S. Swiny, R.L. Hohlfelder, H.W. Swiny, eds.), Atlanta, 339-356.

WEDDE, M. (1999): War at sea: The Mycenaean and Early Iron Age Oared Galley, Polemos. II. Le contexte guerrier en Égée à l'âge du Bronze. 7e rencontre égéenne internationale (Liège 1998) (R. Laffineur ed.), Liège, 465-476.

WEDDE, M. (2000): Towards a Hermeneutics of Aegean Bronze Age Ship Imagery, Mannheim-Möhnesse.

YAMADA, K. (2005): 'From the Upper Sea to the Lower Sea'. The Development of the Names of Seas in the Assyrian Royal Inscriptions, Orient, Report of the Society for Near Eastern Studies in Japan 40, 31-55. 